Rakenteiden Mekaniikka (Journal of Structural Mechanics)

Vol. 50, No 3, 2017, pp. 137 - 140

https://rakenteidenmekaniikka.journal.fi/index

https://doi.org/10.23998/rm.64918

(c) The author(s) 2017.

Open access under CC BY-SA 4.0 license.

\title{
Stiffness and strength of a semi-regular lattice
}

\author{
Tomas Their and Luc St-Pierre ${ }^{1}$
}

Summary. Honeycombs and other lattice materials have the advantage that their topology can be designed to achieve unique combinations of properties, such as high strength at low density. The work presented here is exploratory in nature: we investigated the mechanical properties of the two-dimensional semi-regular snub square lattice and compared its performances to other topologies. Analytical expressions for the uniaxial stiffness and compressive strength were developed and validated against Finite Element simulations. The results showed that the snub square lattice is stiffer and stronger than the diamond lattice, and has a higher resistance to elastic buckling than the triangular lattice.

Key words: lattices, tesselations, buckling, finite element method

Received 15 June 2017. Accepted 16 July 2017. Published online 21 August 2017

\section{Introduction}

Lattice materials are interconnected arrays of struts, and they possess a major advantage over conventional materials: their architecture can be designed to achieve unique combinations of properties, such as high strength at low density [1]. A few examples of two-dimensional lattices are given Fig. 1a-c; these can be categorised as regular, semiregular or other. Regular lattices are made by tessellating a regular polygon; hence, there are only three possibilities: triangular, square and hexagonal. Semi-regular tessellations, like the Kagome lattice, are generated using two or more regular polygons. It can be shown that there are only eight semi-regular combinations possible [2]. Finally, other topologies, such as the diamond lattice, can be constructed using two or more polygons of different sizes.

The mechanical properties of lattices are governed by three parameters: its topology, the properties of the parent material and its relative density $\bar{\rho}$. The latter is defined as the volume fraction of solid material, and can be expressed as a function of the strut thickness $t$ and length $l$ according to:

$$
\bar{\rho}=A\left(\begin{array}{l}
t \\
l
\end{array}\right)
$$

\footnotetext{
${ }^{1}$ Corresponding author. luc.st-pierre@aalto.fi
} 

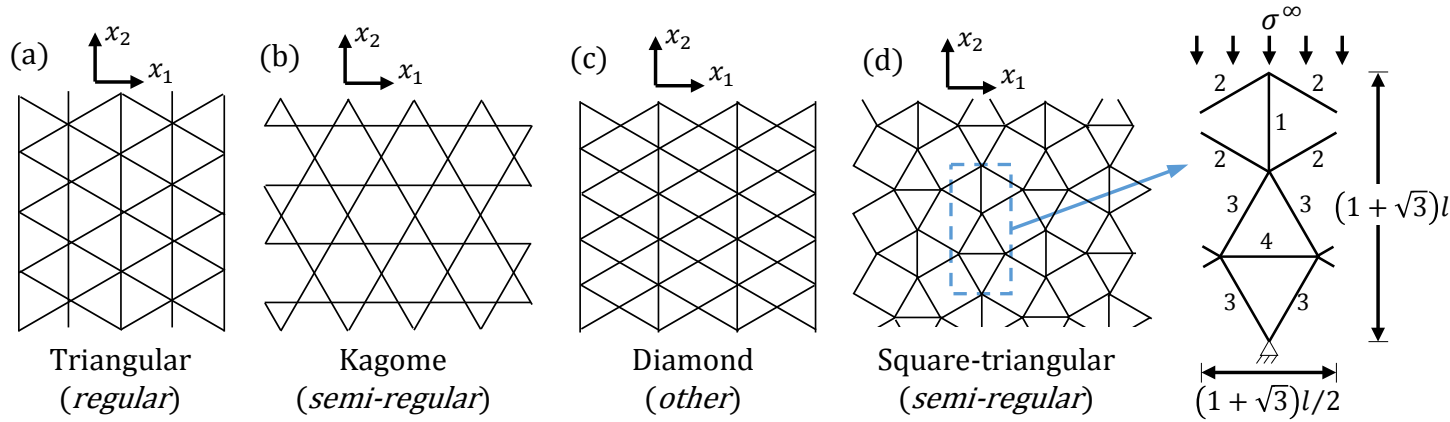

Figure 1. Examples of different lattice topologies: (a) triangular, (b) Kagome, (c) diamond and (d) snub square.

where the constant $A$ depends on the topology, see Table 1. Similarly, the lattice's elastic stiffness $\bar{E}$, elastic buckling strength $\bar{\sigma}_{e l}$ and yield limit $\bar{\sigma}_{y}$ can be expressed as:

$$
\bar{E}=B \bar{\rho} E_{s}, \quad \bar{\sigma}_{e l}=C \bar{\rho}^{3} E_{s} \quad \text { and } \quad \bar{\sigma}_{y}=D \bar{\rho} \sigma_{y s},
$$

respectively, where the Young's modulus $E_{s}$ and yield strength $\sigma_{y s}$ are the properties of the cell wall material. Values of $B, C$ and $D$ are also included in Table 1 for compression along the $x_{2}$ direction. Comparing these coefficients reveals that the Kagome lattice offers the best performances, mainly due to its high elastic buckling strength (coefficient $C$ ).

The Kagome lattice is a semi-regular tessellation and its high stiffness and strength are encouraging enough to justify investigating the properties of other semi-regular morphologies, such as the snub square lattice shown in Fig. 1d. Therefore, the objective of this study is to derive the value of $A, B, C$ and $D$ for the snub square lattice and compare its performances to those of existing architectures.

\section{Analytical modelling}

The snub square lattice can be constructed by tessellating the unit cell shown in Fig.1d and its relative density is given by:

$$
\bar{\rho}=\frac{20}{(1+\sqrt{3})^{2}}\left(\frac{t}{l}\right) \approx 2.68\left(\frac{t}{l}\right) .
$$

With a simple structural analysis, it is possible to express the force in each strut of the lattice $F_{i}$ as a function of the remote nominal stress $\sigma^{\infty}$ as:

Table 1. Coefficients for the relative density, stiffness, elastic buckling strength and yield limit.

\begin{tabular}{llllll}
\hline Topology & $A$ & $B$ & $C$ & $D$ & Reference \\
\hline Triangular & $2 \sqrt{3}$ & $1 / 3$ & 0.0914 & $1 / 3$ & {$[1,3]$} \\
Kagome & $\sqrt{3}$ & $1 / 3$ & 0.366 & $1 / 3$ & {$[1,3]$} \\
Diamond & $5 / \sqrt{3}$ & $1 / 5$ & 0.0789 & $1 / 5$ & {$[3]$} \\
Snub square & $20 /(1+\sqrt{3})^{2}$ & 0.280 & 0.125 & 0.273 & this work \\
\hline
\end{tabular}




$$
F_{1}=\frac{(1+\sqrt{3})}{2} \sigma^{\infty} b l, \quad F_{2}=0 \quad \text { and } \quad F_{3}=-F_{4}=\frac{(3+\sqrt{3})}{6} \sigma^{\infty} b l,
$$

where $b$ is the depth of the lattice (in the out-of-plane direction). The nominal compressive strain $\epsilon^{\infty}=4 \sigma^{\infty} l /\left(3 E_{s} t\right)$ can be obtained using the principle of virtual work and it follows that the lattice's stiffness $\bar{E}$ is given by:

$$
\bar{E}=\frac{\sigma^{\infty}}{\epsilon^{\infty}} \Rightarrow \frac{\bar{E}}{E_{s}}=\frac{3}{4}\left(\frac{t}{l}\right)=\frac{3(1+\sqrt{3})^{2}}{80} \bar{\rho} \approx 0.280 \bar{\rho} .
$$

The elastic buckling strength of the lattice is derived by equating the force in the most loaded member $\left(F_{1}\right)$ to the Euler buckling load for a built-in strut, which gives:

$$
F_{1}=\frac{\pi^{2} E_{s} b t^{3}}{3 l^{2}} \Rightarrow \frac{\bar{\sigma}_{e l}}{E_{s}}=\frac{2 \pi^{2}}{3(1+\sqrt{3})}\left(\frac{t}{l}\right)^{3} \approx 0.125 \bar{\rho}^{3} .
$$

Otherwise, the yield limit of the lattice is obtained when the stress in beam 1 reaches the yield strength $\sigma_{y s}$, and this returns:

$$
F_{1}=\sigma_{y s} b t \Rightarrow \frac{\bar{\sigma}_{y}}{\sigma_{y s}}=\frac{2}{(1+\sqrt{3})}\left(\frac{t}{l}\right)=\frac{(1+\sqrt{3})}{10} \bar{\rho} \approx 0.273 \bar{\rho} .
$$

These analytical relations were validated against Finite Element (FE) simulations, and the model developed is described in the next section.

\section{Description of the Finite Element model}

All calculations were performed with the implicit solver of the commercial software Abaqus. The cell walls were assumed to be made from a linear elastic perfectly plastic solid characterised by a Young's modulus $E_{s}=200 \mathrm{GPa}$, a Poisson's ratio $\nu=0.3$ and a yield strength $\sigma_{y s}=200 \mathrm{MPa}$.

The simulations were done on the unit cell shown in Fig. 1d: periodic boundary conditions were imposed such that the nominal compressive strain $\epsilon_{22}^{\infty}$ was prescribed whereas natural (stress free) boundary conditions were imposed in the $x_{1}$ direction. Each strut of the unit cell was discretised using 50 Timoshenko beam elements. Note that since the slenderness ratio $l / t$ is greater than 10 in all cases, the struts behaved essentially as Euler-Bernoulli beams, which is consistent with the assumptions of our analytical model.

A small geometric imperfection was introduced in the FE model. The perturbation affected the entire geometry and had the shape of the first buckling mode (obtained from an eigenvalue buckling analysis) with an amplitude of $0.01 t$. Note that the simulations were relatively imperfection insensitive: modifying its shape to a combination of buckling modes and changing its amplitude within $0.001 t-0.05 t$ had a minor effect on the results.

\section{Results and discussion}

The analytical and FE predictions are compared in Fig. $2 \mathrm{a}$ and $\mathrm{b}$ for the normalised stiffness and normalised compressive strength, respectively. The compressive strength is the minimum value between the elastic buckling stress and the yield limit, i.e. $\bar{\sigma}=$ $\min \left(\bar{\sigma}_{e l}, \bar{\sigma}_{y}\right)$. In general, there is a good agreement between $\mathrm{FE}$ and analytical results, which indicates that Eqs. (5-7) are accurate. 

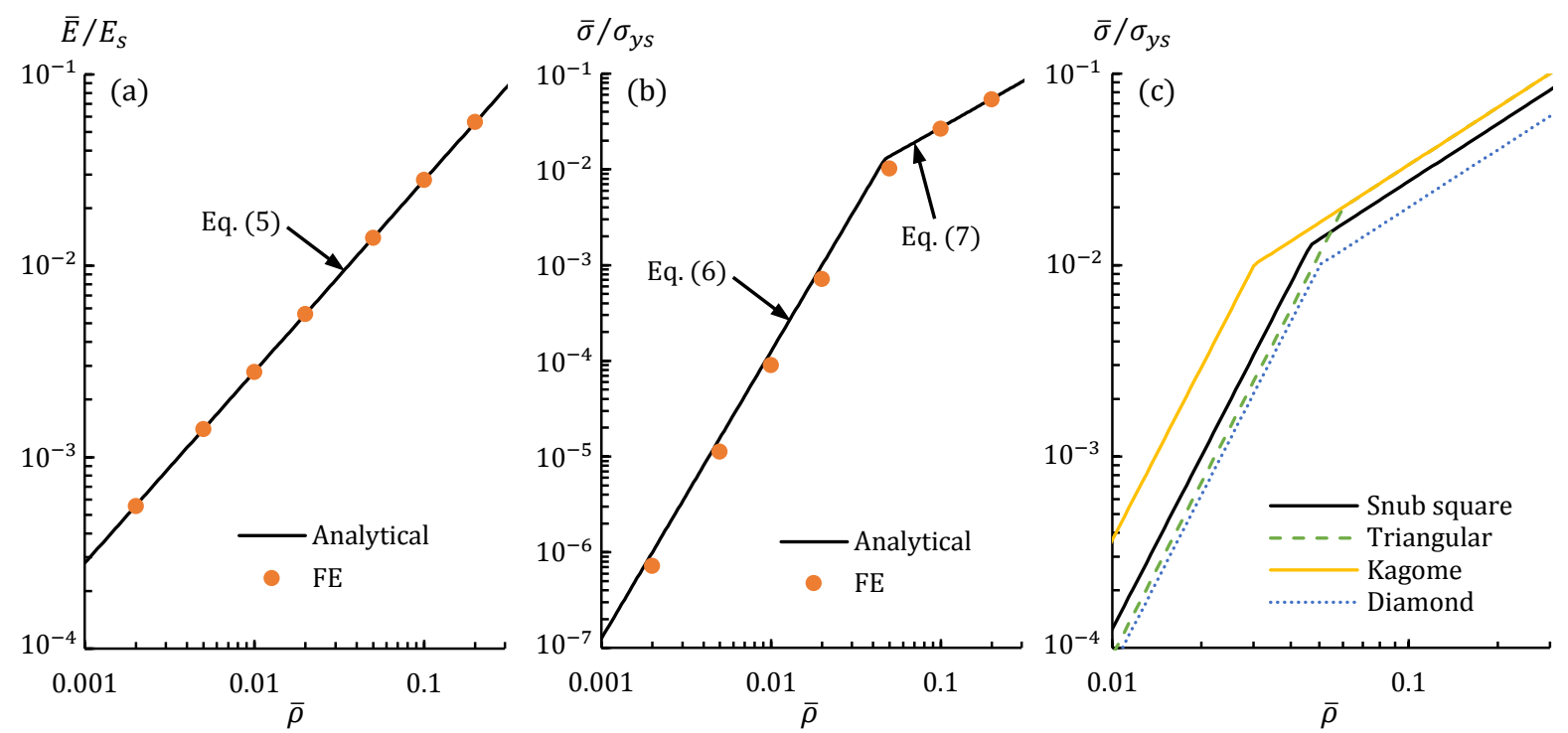

Figure 2. Comparison between analytical and FE results for the snub square lattice: (a) normalised stiffness and (b) normalised compressive strength as a function of relative density $\bar{\rho}$. The normalised compressive strength of different lattices is compared in part (c).

A comparison with other topologies reveals that the snub square lattice is softer than triangular and Kagome architectures, but stiffer than the diamond lattice, see Table 1. The normalised strength of these lattices is compared in Fig. 2c: the results show that the snub square lattice is stronger than diamond and also outperforms the triangular lattice for $\bar{\rho}<0.06$ because of its high elastic buckling strength.

\section{Conlusions and future work}

Analytical relations for the stiffness and compressive strength of the snub square lattice were derived and validated against FE simulations. The performances of the snub square lattice were found to be inferior to those of the Kagome lattice, but superior to the ones of the diamond tessellation. An experimental validation is currently underway while the response of the snub square lattice in shear remains to be investigated.

\section{References}

[1] N.A. Fleck, V.S. Deshpande and M.F. Ashby. Micro-architectured materials: past, present and future. Proceedings of the Royal Society A, 466:2495-2516,2010. https: //doi.org/10.1098/rspa.2010.0215

[2] H.M. Cundy and A.P. Rollett Mathematical models. Tarquin, St. Albans, UK, 1981.

[3] A.-J. Wang and D.L. McDowell. In-plane stiffness and yield strength of periodic metal honeycombs. Journal of Engineering Materials and Technology, 126(2):137-156,2004. https://doi.org/10.1115/1.1646165

Tomas Their, Luc St-Pierre

Aalto University, Department of Mechanical Engineering

PO Box 14300, FI-00076

tomas.their@aalto.fi, luc.st-pierre@aalto.fi 